\title{
Expression of mannose binding lectin in HIV-I-infected brain: implications for HIV-related neuronal damage and neuroAIDS
}

This article was published in the following Dove Press journal:

Neurobehavioral HIV Medicine

28 May 2011

Number of times this article has been viewed

\author{
Kumud K Singh' \\ Satyanarayana Nathamu' \\ Anthony Adame ${ }^{2}$ \\ Tara U Alire' \\ Wilmar Dumaop ${ }^{2}$ \\ Ben Gouaux ${ }^{3}$ \\ David J Moore ${ }^{3}$ \\ Eliezer Masliah ${ }^{2}$ \\ and HIV Neurobehavioral \\ Research Center Group \\ 'Department of Pediatrics, \\ ${ }^{2}$ Department of Neurosciences, \\ ${ }^{3}$ Department of Psychiatry, \\ University of California San Diego, \\ La Jolla, CA, USA
}

\begin{abstract}
Mannose binding lectin (MBL) activates complement pathway that leads to pathogen opsonization and phagocytosis. MBL deficiency is linked to HIV transmission and disease progression. We sought to determine the role of MBL in HIV encephalitis (HIVE) by evaluating its presence and distribution in the HIV-1-infected brain and by assessing its association with monocyte chemoattractant protein-1 (MCP-1) expression. This retrospective study utilized archived post-mortem brain tissues obtained from 35 individuals enrolled in a longitudinal study as part of the California NeuroAIDS Tissue Network. MBL, MCP-1 and brain cell markers in post-mortem brain tissues with or without HIVE were evaluated using immunocytochemistry, immunofluorescence, confocal microscopy, and western blots. MBL was expressed in neurons, astrocytes, microglia, and oligodendrocytes of the frontal cortex of the HIV-1-infected brain. Overall, there were $30 \%$ to $40 \%$ more MBL-positive brain cells in HIVE vs non-HIVE cases ( $P=0.01$, paired $t$-test). Specifically, there was an increased MBL expression in the neuronal axons of HIVE cases. Also, western blots showed 3- to 4-fold higher levels of $78 \mathrm{kD} \mathrm{MBL}$ trimers in HIVE vs non-HIVE cases. This MBL-HIVE link was further confirmed by MBL associated higher MCP-1 expression in HIVE vs non-HIVE cases. HIV negative healthy individuals and normal or the gp 120 transgenic mice did not show any differential MBL expression. Increased MBL expression in the major brain cell types, specifically in the neuronal axons of HIVE brain, and MBL associated higher MCP-1 expression in HIVE suggest that MBL could cause neuroinflammation and neuronal injury through MBL complement activation pathway. Keywords: mannose binding lectin, HIV encephalitis, complement activation, neuroinflammation, neuroAIDS, MCP-1
\end{abstract}

\section{Introduction}

Innate immunity refers to antigen-nonspecific defense mechanisms that a host uses immediately or within several hours after exposure to an antigen. Unlike adaptive immunity, innate immunity does not recognize every possible antigen. Instead, it is designed to recognize a few highly conserved structures present in many different microorganisms. The mannose-binding lectin (MBL), also called mannose-binding protein, coded by the $M B L 2$ gene, is an acute-phase protein that is synthesized by the liver and is released into the bloodstream where it recognizes and binds to mannose residues or carbohydrates on pathogens such as bacteria, yeast, viruses, or parasites. ${ }^{1-3}$ Binding activates the lectin complement pathway via MBL-associated serine proteases (MASPs),${ }^{4}$ which results in opsonization of pathogens, chemotaxis and activation of leukocytes, and direct killing of pathogens. Among 3 pathways of complement activation, alternate pathway directly activates complement component $\mathrm{C} 3$ while 
both classical and lectin pathways cleave $\mathrm{C} 4$ - the former by binding of $\mathrm{Clq}$ to immune complexes and the latter by the binding of MBL to molecular entities expressing appropriate sugar patterns. MBL deficiency was initially recognized as an opsonic defect in children ${ }^{5-7}$ with frequent unexplained infections and was linked to increased severity and incidence of complications for several inherited immunodeficiency and autoimmune diseases. ${ }^{8,9}$

MBL belongs to the family of collectins. ${ }^{10}$ The collectins have a dual function: one is to bind specifically to carbohydrate structures on the surface of a pathogen, the other is subsequently to recruit other cells and molecules to destroy the pathogen. ${ }^{10}$ After binding of the collectins to the microbial surface, effector mechanisms such as agglutination, neutralizing or opsonization of the microorganisms for phagocytosis are initiated. In the case of MBL the opsonization can be further enhanced by MBL-mediated complement activation pathway.

The $32 \mathrm{kDa}$ subunits coded by the $M B L 2$ gene are reorganized in structural units or 'monomers' which further associate to form high molecular weight (MW) MBL oligomers. Only the high MW oligomer structure is capable of activating complement. ${ }^{1,2}$ Whereas only one form was identified in humans, chimpanzees, and chickens, two forms of MBL, MBL-A and MBL-C, were characterized in rodents, rabbits, bovine, and rhesus monkeys. ${ }^{11}$ The liver is the major site of expression for both MBL genes and lower copy numbers were found in kidney, spleen, muscle, and brain. ${ }^{12}$ The role of MBL in cerebrum development has also been reported. ${ }^{13}$

MBL recognizes mannose $\mathrm{N}$-linked glycan residues of HIV-1 gp41/120 and elicits complement activation, cytokine responses, and macrophage-mediated HIV-1 opsonization. ${ }^{14}$ During HIV-1 infection of the brain and in cases of HIV-associated dementia, an accumulation of HIV-1 gp41 has been observed. ${ }^{15}$ Additionally, immune complex deposits have been identified in the choroid plexus of patients with acquired immune deficiency syndrome (AIDS). ${ }^{16}$ MBL-mediated complement activation is observed in Neisseria meningitidis, ${ }^{17}$ and MBL deficiency related to the presence of variant $M B L 2$ alleles has been implicated in the risk of meningococcal disease, ${ }^{18} \mathrm{HSV}-2,{ }^{19}$ and HIV-1 infections. ${ }^{14,20,21}$ We have shown earlier that the presence of $M B L 2$ genetic variants affecting its function and expression are associated with more rapid progression to CNS impairment. ${ }^{22,23}$

In the current study, we show that: i) MBL is expressed in the major cell types of the HIV-1 infected brain; ii) MBL expression is increased in the neuronal axons of
HIV-1 infected brain with HIV encephalitis (HIVE); and iii) higher MBL expression is associated with increased MCP-1 in the HIVE cases. Taken together, our results suggest that MBL expression is increased in concurrence with MCP-1 during HIVE, suggesting a potential association of MBL-mediated complement activation with neuroinflammation and neuronal injury in HIV infected brain.

\section{Methods}

\section{Characteristics of the clinical samples}

A total of 35 individuals enrolled in a longitudinal study as part of the California NeuroAIDS Tissue Network coordinated at the UC San Diego HIV Neurobehavioral Research Center $^{24}$ were selected for the present study. Most patients died as a result of acute bronchopneumonia and/or septicemia and the autopsy was performed within 24 to 36 hours of death. Of the 35 studied subjects from whom the postmortem brain tissues were evaluated for these studies, 16 were non-HIVE and HIVE each and 3 were normal HIV negative healthy individuals at the time of death. Of these, 24 were non-Hispanic and 11 were Hispanic. Of the 24 nonHispanic, 20 were Whites, 3 were African Americans, and 1 was a Pacific Islander. Median age was 43 years and $83 \%$ $(29 / 35)$ were male. Fifty-one percent of subjects included in this study were seen for antemortem neuromedical and neuropsychological examinations. The remaining 49\% of subjects were enrolled in the study for autopsy only. Among those who were seen for antemortem examinations, $48 \%$ were on antiretroviral therapy at the time of their last assessment, and $74 \%$ had taken at least one antiretroviral drug at some point in the past. Despite the use of antiretrovirals and their potential effects on neuropathogenesis, diagnostic characteristics of HIV encephalitis were observed in all HIVE brain tissues. Among subjects with CD4 data available $(n=22)$, the median CD4 count was 56 (IQR 11-188) and did not differ by HIVE status $(P=0.16)$. As expected, the rate of virologic suppression was significantly higher in the nonHIVE group (58\% in plasma, 53\% in cerebrospinal fluid) than the HIVE group ( $0 \%$ in plasma, $0 \%$ in cerebrospinal fluid) ( $\mathrm{n}=20, P=0.01$ and $\mathrm{n}=24, P<0.01$, respectively). Post-mortem brain tissues from age-matched individuals were used for this study. Information obtained from available neuropathological data was used to exclude cases with anoxic brain injury or with CNS opportunistic infections at the time of death. After macroscopic examination, tissue blocks from the right midfrontal cortex were obtained and immersionfixed for 48 hours at $4^{\circ} \mathrm{C}$ in $4 \%$ paraformaldehyde. For immunocytochemical analysis, samples were serially sectioned at 
$40 \mu \mathrm{m}$ with the Vibratome 2000 (Leica, Deerfield, IL). The diagnosis of HIVE was based on the presence of mononuclear giant cells (MNGC), HIV-infected microglial cells and viral load as described earlier. ${ }^{25,26}$ Histopathologic evidence of MNGC, microgliosis, and myelin pallor in fronto-temporal cortex, hippocampus, basal ganglia, midbrain, and cerebellum was confirmed by visual inspection that revealed tissue changes typical of HIVE. Neuronal damage was assessed in MAP2-immunolabeled vibratome sections and defined as less than $19 \%$ of the midfrontal cortex neuropil covered by MAP2-immunoreactive dendrites. ${ }^{27}$ It has been previously shown that while in well-characterized control individuals, MAP2 values were usually above 19\%, individuals with HIVE displaying cognitive impairment had values below $19 \%$ indicating significant dendritic loss. ${ }^{27}$ The tissues were acquired via the California NeuroAIDS Tissue Network which has approval from the UCSD institutional research board to collect and utilize postmortem tissues from HIV positive individuals.

\section{Immunofluorescence analysis of $M B L$ and MCP-I in post-mortem brain tissue}

Tissue sections from the frontal cortex of healthy HIV negative, HIVE and non-HIVE human brain samples were analyzed for the localization of MBL in microglia, astrocytes, oligodendrocytes, and neurons. Paraffinized tissue sections were incubated at $65^{\circ} \mathrm{C}$ for 1 hour, washed with citrisolve clearing agent (Catalog \# 22143975, Fischer Scientific, Pittusburgh, PA) and rehydrated in decreasing concentrations of ethanol (50:50 citrisolve and ethanol, 100\%, 95\%, 70\%, $50 \%, 20 \%$ ethanol, milliQ water, and phosphate buffer saline, PBS). Sections were treated with permeabilization buffer containing saponin (Catalog \# PB001, Invitrogen, Carlsbad, CA) for 5 minutes at room temperature followed by heat induced epitope retrieval in $10 \mathrm{mM}$ sodium citrate buffer, pH 6.0 (Catalog \# AP9003-500, Thermo Scientific, Rockford, IL). For epitope retrieval, tissue sections were placed in a $650 \mathrm{~W}$ microwave oven and heated intermittently for 15 minutes and allowed to cool to room temperature. Sections were washed with PBS for 20 minutes and nonspecific sites were blocked in 5\% normal goat serum with $5 \%$ bovine serum albumin (BSA) in PBS for 1 hour at room temperature. $^{28}$

Rabbit polyclonal anti-MBL2 (Catalog \# HPA002027, Sigma-Aldrich, St. Louis, MO) and one of the brain cell markers, mouse monoclonal microtubule associated protein 2 (MAP2) for neurons, mouse monoclonal CD68 for microglia, mouse monoclonal glial fibrillary acidic protein (GFAP) for astrocytes, mouse monoclonal myelin oligodendrocyte glycoprotein (MOG) for oligodendrocytes (Catalog numbers Ab4648, Ab845, Ab4648, Ab24022 respectively from Abcam, Cambridge, MA) were diluted 1:200 in PBS with $1 \% \mathrm{BSA}$ and were incubated overnight at $4^{\circ} \mathrm{C}$. After washing for 20 minutes with PBS, sections were incubated in the dark for an hour with goat anti-rabbit IgG conjugate Alexa Fluor 488 (Catalog \# A11008, Invitrogen, Carlsbad, CA) for probing MBL antibody and donkey anti-mouse IgG conjugate Alexa Fluor 594 (Catalog \# A21203, Invitrogen, Carlsbad, CA) for any of the markers among MAP2, CD68, GFAP, MOG respectively. After incubation of secondary antibodies, sections were washed for 20 minutes in PBS and mounted with Prolong Gold anti-fade reagent (Catalog \# P36931, Invitrogen, Carlsbad, CA) containing DAPI (4', 6-diamidino-2-phenylindole) and covered with a cover slip. Mounted sections were air-dried overnight and analyzed by confocal microscope. In each case, control experiments were conducted with IgG isotope, serum, primary or secondary antibody alone.

Appropriate care was taken to avoid the cross-reactivity and nonspecific binding of antibodies. We used confocal microscopy (Olympus FV1000) to capture emission wavelength of individual fluorescent dyes. Fluorescent dyes used, such as DAPI (358 nm excitation and $461 \mathrm{~nm}$ emission) for nuclei, Alexa Fluor 488 (495 nm excitation and $519 \mathrm{~nm}$ emission) for MBL, and Alexa Fluor 594 (590 nm excitation and $617 \mathrm{~nm}$ emission) for cell marker have significant gaps between the emission wavelengths ruling out the possibility of cross interference. Consequently, green fluorescence for MBL and red fluorescence for cell markers were individually emitted at their respective wavelengths and when the two signals co-localized, yellow spots were observed. All secondary antibodies were selected from different species (eg, donkey) compared with the primary antibodies (eg, rabbit, mouse) to further eliminate any cross-reactivity between the species in which the antibodies were raised. Also, immunofluorescence staining experiments were repeated several times with different cases to ensure reproducibility of results.

For regular immunohistostaining of MBL in postmortem brain tissue, after antigen retrieval and nonspecific sites blocking by serum, rabbit anti MBL polyclonal primary antibody was applied (1:200) and incubated overnight at $4^{\circ} \mathrm{C}$. Sections were developed in biotinylated anti-rabbit secondary antibody, followed by Avidin D-HRP (ABC Elite) and reacted with 3,3'-diaminobenzidine (DAB, $0.2 \mathrm{mg} / \mathrm{mL})$ in $50 \mathrm{mM}$ Tris buffer $(\mathrm{pH} 7.4)$ with $0.001 \%$ 
hydrogen peroxide. After washing with water, slides were counter-stained with 50\% hematoxylene and dehydrated by ethanol and citrisolv, washed with water, air dried overnight and mounted with one drop of entellan on the tissue and coverslip. Biotinylated anti-rabbit secondary antibody and other reagents were obtained from Vector Laboratories (Burlingame, CA).

For MCP-1 related immunofluoresence analyses, mouse monoclonal anti MCP-1 primary antibody (Catalog \# Ab9858, Cambridge, MA) and donkey anti-mouse IgG conjugate Alexa Fluor 594 secondary antibody were used.

\section{Confocal microscopy}

The immunostained sections were visualized with Olympus FV1000 Confocal Microscope with appropriate excitation/ emission filter pairs (403 nm laser window 410-483 nm, $488 \mathrm{~nm}$ laser window 493-538 nm, $543 \mathrm{~nm}$ laser window 548-628 nm). Images were collected using the microscope in sequential plane mode with an average of 4 and a format of $1024 \times 1024$ pixels using X100 oil immersion lens. Images were exported to Olympus FV1000 Viewer software Ver.02.00 to generate the figures. Immunofluorescence of the positive cells co-localized with MBL and respective cell markers were counted in 5 areas of a section (4 corner areas and 1 central area of a section in at least 4 slides). Percent increase for the expression of MBL from HIV to HIVE cases was calculated and $P$ value was determined using the 2-tailed paired $t$-test.

\section{Detection of viral p24 protein in the post-mortem brain tissue}

Sections from the frontal cortex region were immunostained with goat polyclonal antibody to HIV-1 p24 (Catalog \# 4999-9007, AbD Serotec, Raleigh, NC) to detect its presence in post-mortem brain tissues with or without HIVE.

\section{Detection of MBL expression in normal and transgenic gp 120 mouse model}

Brain tissue sections from 4 each of normal and transgenic (tg) gp120 mice were used to evaluate the expression level of murine MBL proteins MBL-1 and MBL-2 (Catalog \# AF2077 and MAB2208 respectively, R \& D Systems, Minneapolis, MN). The gp120 tg mice were provided by Dr Masliah and were described earlier. ${ }^{29}$ Mixed gender, 12-month-old C57/B16 strain mice were evaluated for these studies. Immunohistostaining protocols used for the mice brain tissue were similar to the MBL detection protocol described previously.

\section{Western blot analyses of MBL} and MCP-I in post-mortem brain tissue

Total proteins extracts from non-HIVE, HIVE and normal postmortem brain tissues were prepared by using an All-Prep kit (Catalog \# 80004, Qiagen Inc. Valencia, $\mathrm{CA}$ ) and quantified by bicinchoninic acid (Catalog \# 23227, Thermo Scientific, Rockford, IL). A total protein of $20 \mu \mathrm{g}$ from each brain tissue was separated on $4 \%$ to 12\% NuPAGE Bis-Tris Gel electrophoresis (Catalog \# NP0322BOX, Invitrogen, Carlsbad, CA). Western blots were transferred and developed with MBL or MCP-1 antibodies that were used for immunofluorescence studies. A mouse monoclonal anti- $\beta$-actin antibody (Catalog \# A2228, Sigma-Aldrich, St Louis, MO) was used as an equal protein loading control and for MBL/MCP-1 quantitation by band intensity normalization using Image J software (NIH, USA). Ponceau stain (Catalog \# P7170-1L, Sigma-Aldrich, St Louis, MO) and Magic Mark XP Western protein standard (Catalog \# LC 5602, Invitrogen, Carlsbad, CA) were used for locating protein bands.

\section{MBL2 genotyping of post-mortem brain tissues}

Total DNA extracted from the frontal cortex of the 3 groups of post-mortem brain tissues (HIV negative healthy, HIVE, non-HIVE) was genotyped for genetic variants in the exons and promoter region using real-time PCR melting curve analyses using a LightCycler 1.0 (Roche Diagnostics Inc., Indianapolis, IN) as described earlier. ${ }^{22}$ Two single nucleotide polymorphisms at promoter positions $-550-\mathrm{G} / \mathrm{C}$ ( $H / L$ variant) and $-221-\mathrm{G} / \mathrm{C}(X / Y$ variant $)$, one in the 5' untranslated region $+4-\mathrm{C} / \mathrm{T}(P / Q$ variant $)$ and 3 genetic variants at codons 52,54 , and 57 in exon 1 at nucleotide positions 223-C/T (Arg52Cys, $A / D$ allele), 230-G/A (Gly54 Asp, $A / B$ allele), and 239-G/A (Gly57Glu, $A / C$ allele), respectively were studied.

\section{Results}

MBL immunoreactivity is present in association with microglia, neurons, astrocytes, and oligodendrocytes in post-mortem brain tissues with HIV encephalitis

First, we used antibodies against MBL and brain cell markers to evaluate the presence of MBL in HIV-1 infected brain. In the frontal cortex of the post-mortem brain tissue from HIV-1 infected individuals, MBL displayed immunoreactivity in the major brain cell types - neurons, astrocytes, microglia, 
and oligodendrocytes (Figure 1). There was a co-localization of MBL and individual brain cell markers. Additionally, DAPI stain clearly marked the nuclear DNA showing that cells with intact nuclei were studied.

\section{Increased MBL expression in post-mortem brain tissue with HIV encephalitis}

To study whether MBL was differentially expressed in different brain cell types, a comparison of MBL expression in microglia, astrocytes, neurons, and oligodendrocytes in non-HIVE, HIVE, and healthy HIV negative cases was done. Overall, there were $30 \%$ to $40 \%$ higher MBL positive brain cells in HIVE vs non-HIVE cases (statistically significant at $P<0.01$, paired $t$-test) (Figure 2). Frontal cortex brain tissues from HIV negative, healthy individuals did not show any significant MBL expression (Figure 2).

\section{Increased MBL expression in neuronal axons of post-mortem brain tissue with HIV encephalitis}

To determine if MBL expression in neuronal axons correlate with the risk of encephalitis, frontal cortex brain tissue sam-
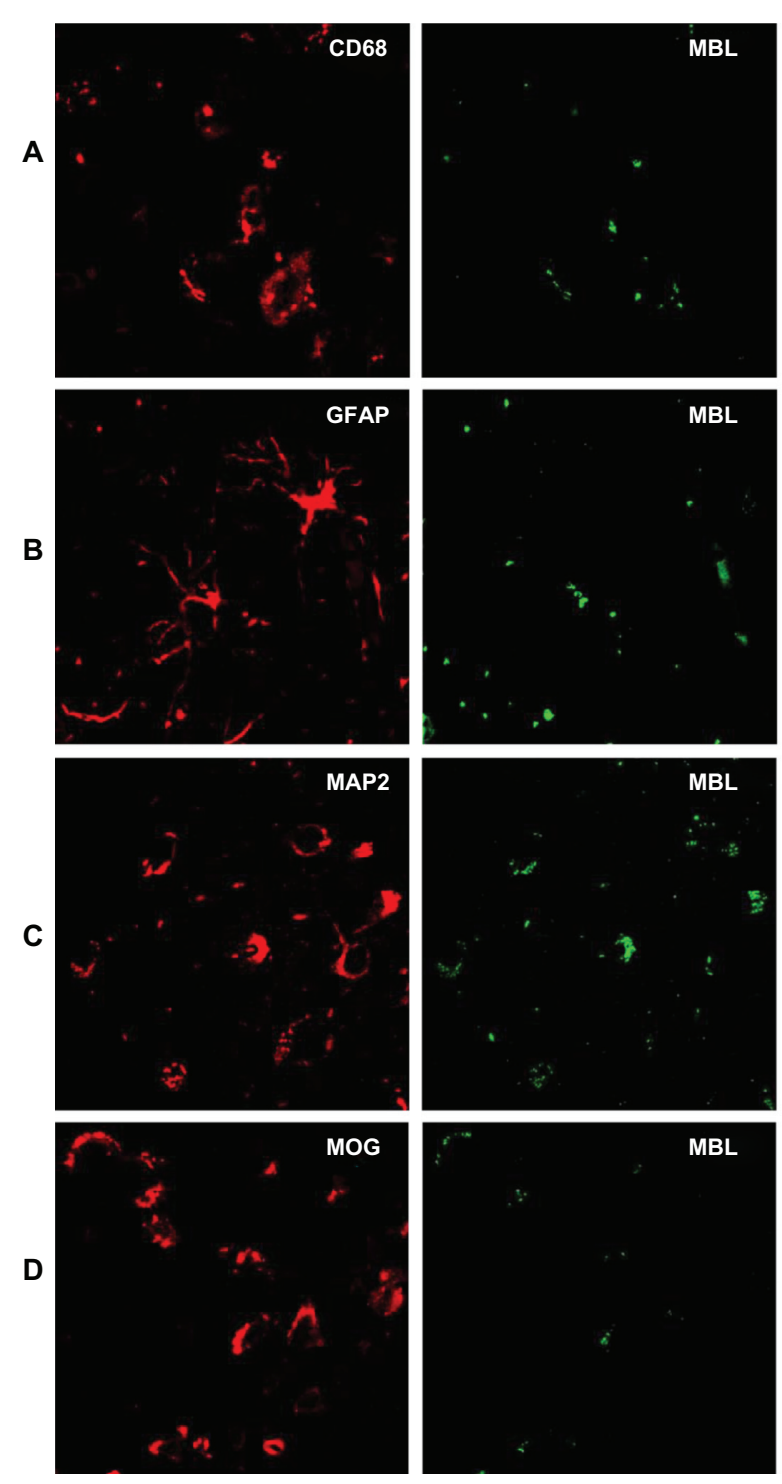
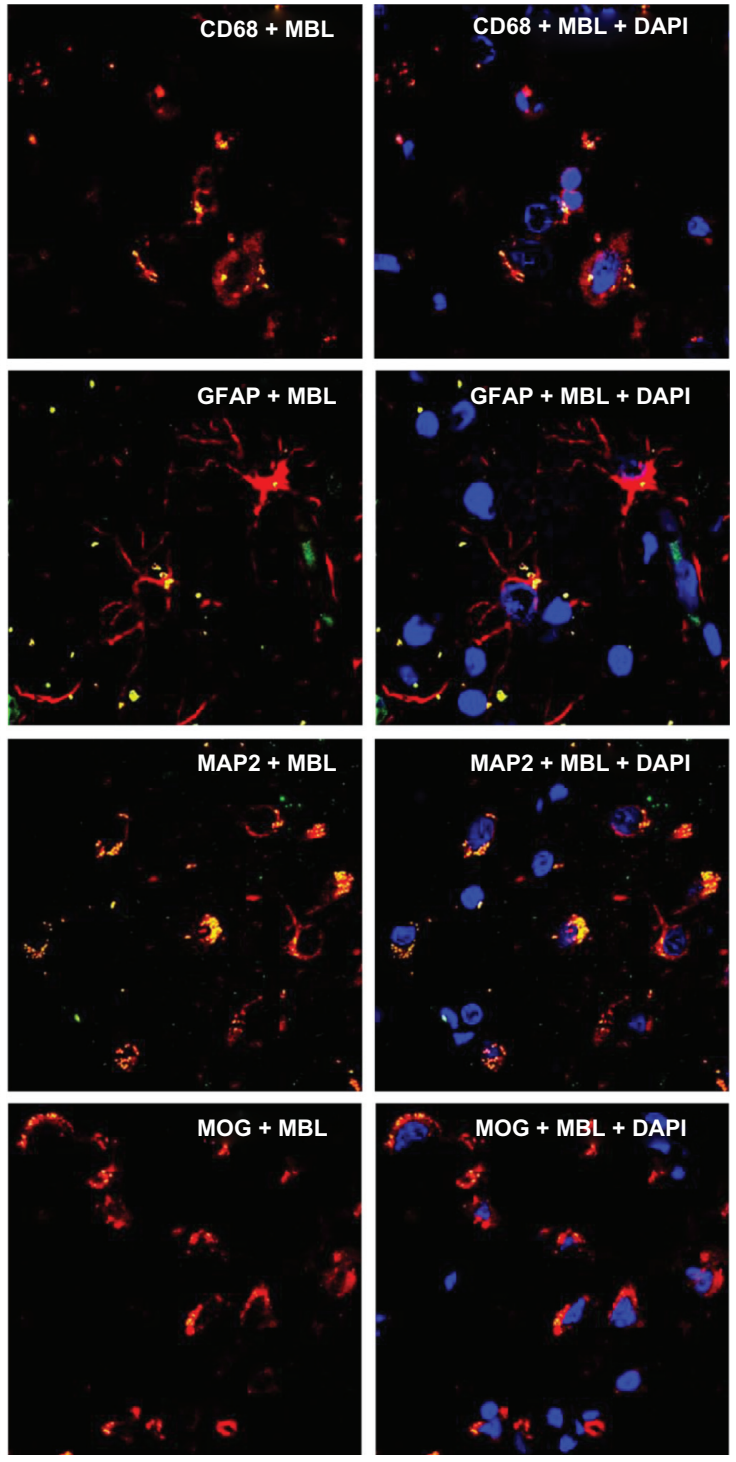

Figure I Distribution and co-localization of MBL and brain cell markers using double immunofluorescence and confocal microscopy at I20 $\mu \mathrm{m}$ scale and I00 $\times$ magnification. Panels A, B, C, and D show mannose binding lectin (MBL) and cell markers for microglia (CD68), astrocytes (GFAP), neurons (MAP2), and oligodendrocytes (MOG) respectively. Primary antibodies were detected with fluorescent Alexa Fluor secondary antibodies. Green fluorescence represents $M B L$, red represents individual brain cell marker, and the yellow fluorescence represents the co-localization of MBL and the respective cell marker. Blue fluorescence represents the presence of DNA in an intact nucleus stained by 4', 6-diamidino-2-phenylindole (DAPI). 


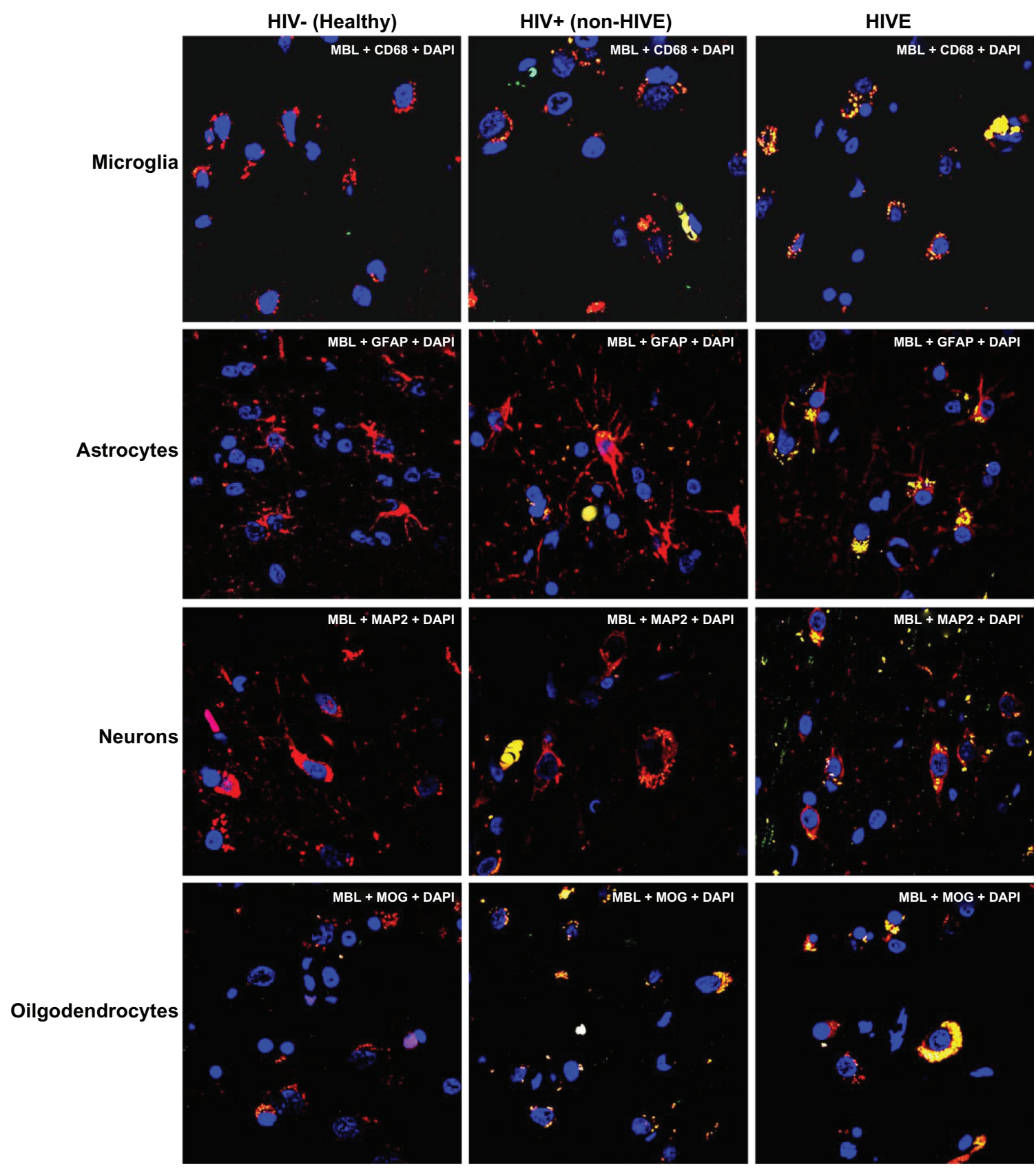

Figure 2 Comparison of mannose binding lectin (MBL) distribution in microglia, astrocytes, neurons, and oligodendrocytes in the post-mortem brain tissues from HIVnegative healthy, HIV+ non-HIVE, and HIVE cases. Methods, scale, magnification, and fluorescence colors are same as in Figure I.

ples from non-HIVE and HIVE cases were immunostained with MBL antibodies. MBL was moderately expressed in nonHIVE (HIV+ cases with no brain alterations) while abundantly expressed in HIVE neuronal axons (Figure 3A, B, G, H). The p24 protein was not notably detected in the non-HIVE cases (Figure 3E) and there was only moderate MBL immunostaining in the axons. However, post-mortem brain tissues from HIVE cases showed abundant p24 staining confirming the presence and multiplication of HIV-1 (Figure 3F).

\section{MBL expression is unchanged in normal and transgenic gp/20 mice}

We analyzed the expression of MBL in normal (nontransgenic) mice and compared these with the gp120 transgenic mice (in Dr Masliah's lab) anticipating that gp120 transgenic mice will probably express higher MBL because of the interaction of MBL with mannose residues on the gp120 protein. However, we did not observe any significant difference in MBL expression in the brain tissue from 

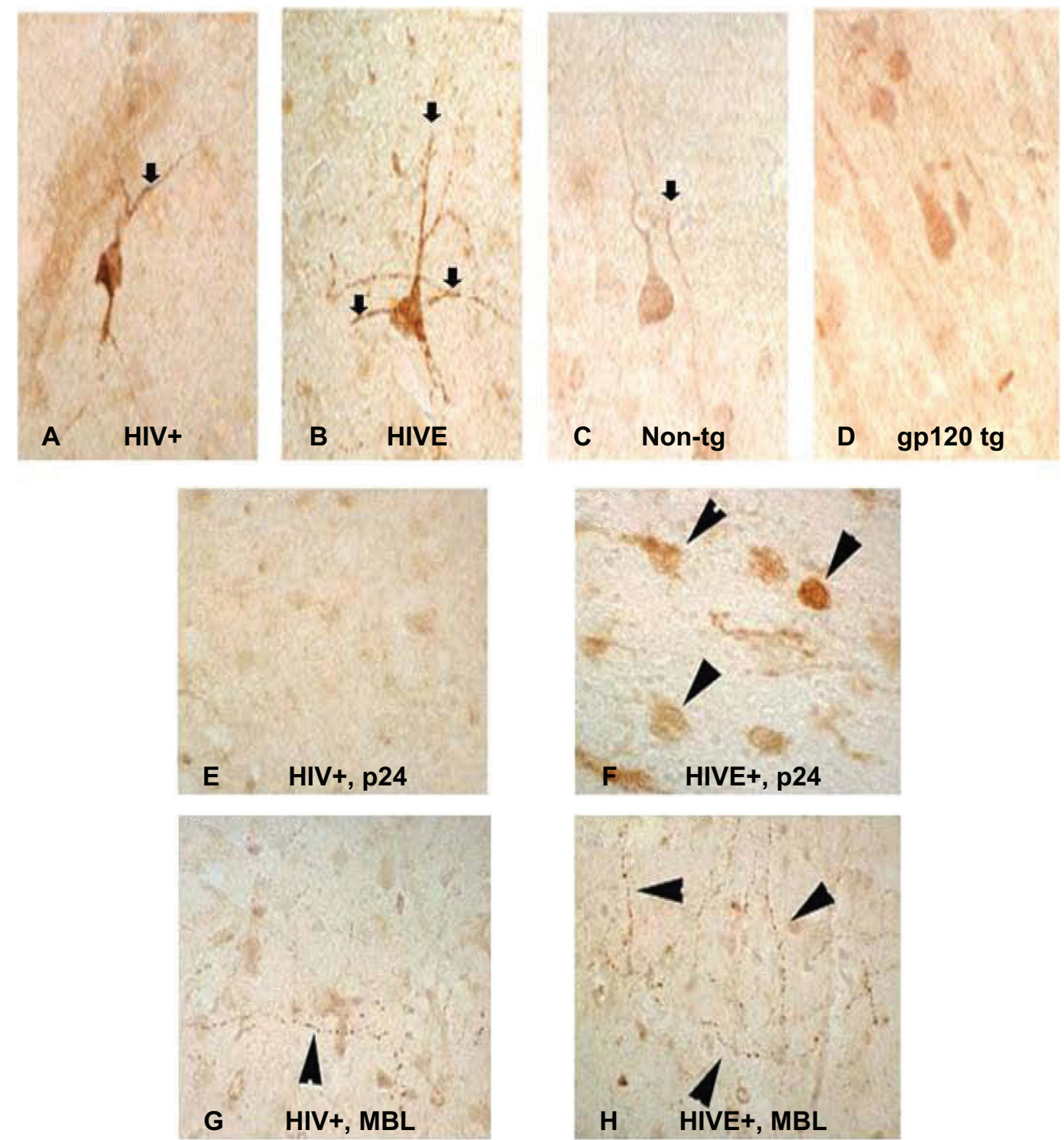

Figure 3 Distribution of mannose binding lectin (MBL) in neuronal axons in human and mice brain tissues. Panels show MBL expression in single neuronal axon in postmortem brain tissues without (A) or with HIVE (B); while a comparative MBL expression in normal nontransgenic mouse (C) and gPI 20 transgenic mouse (D) is shown. Immunoreactive distribution of p24 in microglia of HIV+ non-HIVE (E) and HIVE case (F); and MBL in neuronal axons from HIV+, non-HIVE (G) versus HIVE (H) respectively are shown. Primary antibodies were developed with biotinylated secondary antibody, followed by treatment with Avidin D-HRP and reacted with $3,3^{\prime}$-diaminobenzidine (DAB) in Tris buffer. Slides were counter-stained with 50\% hematoxylene. $120 \mu \mathrm{m}$ scale and I00× magnification was used.

normal mice (Figure 3C) or the gp120 transgenic mice (Figure 3D).

\section{Confirmation of MBL expression in post- mortem brain tissues by western blots}

To corroborate the expression of functional MBL, we determined if MBL trimers were expressed in the HIV-1 infected brain using western blots. Apart from $32 \mathrm{kDa}$ monomers and $51 \mathrm{kDa}$ dimers, $78 \mathrm{kDa}$ trimers of the MBL present in the post-mortem brain tissues were identified (Figure 4A). Using the Image $\mathrm{J}$ software from NIH web site (http://rsb.info.nih.gov/ij/) for quantitation of MBL expressed in 3 groups of brain tissues, we found that MBL expression was 3- to 4-fold higher in the post-mortem brain tissues in the HIVE cases (Figure 4A) compared with those from nonHIVE (statistically significant at $P<0.01$, paired $t$-test) or from HIV negative healthy individuals. Only 2 representative subjects from each group of HIVE, non-HIVE and HIV negative subjects were shown. However, a subject specific pattern of MBL bands was also observed, probably due to the presence of different MBL2 genotypes. MBL antibody used earlier for immunofluorescence and immunohistostaining was 
A
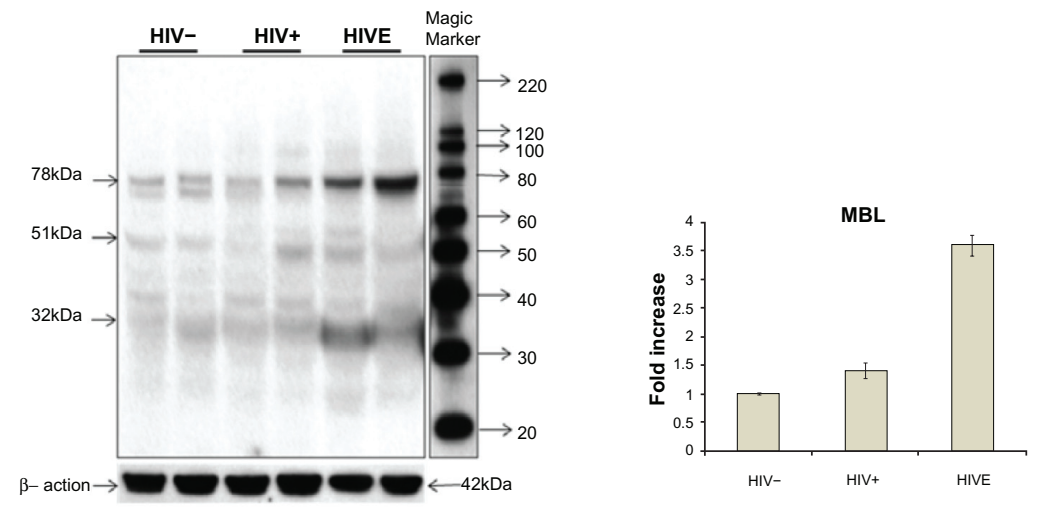

B
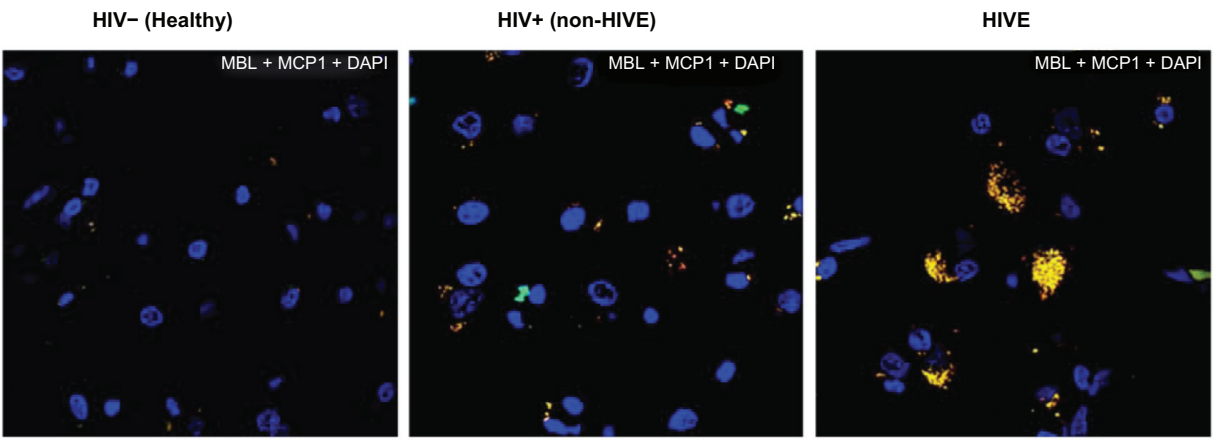

C
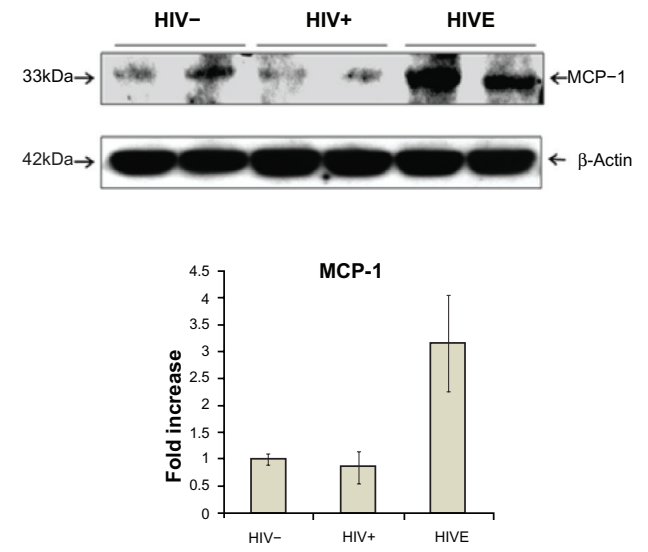

Figure 4 Western blot analyses of mannose binding lectin (MBL) expression in post-mortem brain frontal cortex tissues from two each of HIV-, HIV+ non-HIVE, and HIVE cases (A). $32 \mathrm{kDa}$ monomers, $5 \mathrm{I} \mathrm{kDa}$ dimers and $78 \mathrm{kDa}$ trimers of the MBL were observed. Side panel shows a 3 -fold increase of MBL in HIVE vs HIV+ non-HIVE cases and bars represent the standard deviation of MBL concentration in the studied samples. Distribution of MCP-I and its co-localization with MBL in post-mortem brain frontal cortex tissues using immunofluorescence (B) and western blot (C). Green fluorescence represents MBL, red represents MCP-I, and the yellow fluorescence represents the co-localization of MBL and MCP-I. Blue fluorescence represents the presence of an intact nucleus stained by 4', 6-diamidino-2-phenylindole (DAPI). Method, scale, and magnification are the same as in Figure I. Panel C shows about 3-fold increase of MCP-I in HIVE vs non-HIVE or HIV negative cases. Bars represent the standard deviation of MCP-I concentration in the studied samples.

used for western blots. Beta-actin was used as a housekeeping reference protein and as a loading control for comparing the expression of MBL in the brain tissues.

\section{Increased MCP-I expression in association with MBL in HIV encephalitis}

In order to understand if MBL predicted the MCP-1 accumulation (a marker of HIV-1 related neuroinflammation) in the HIV-1 infected brain, co-localization of MBL and MCP-1 was studied. Increased expression of MCP-1 was observed in HIVE vs non-HIVE or HIV negative cases ( $P=0.01$, paired $t$-test for each comparison) (Figure 4B). Both MBL and MCP-1 were co-localized in the brain suggesting an active role of MBL related MCP-1 accumulation in HIVE. Moreover, western blots from HIVE cases showed an increase of 3-fold in MCP-1 expression 
compared with non-HIVE or healthy HIV negative controls (Figure 4C).

\section{Association of MBL2 genotypes and haplotypes with HIV Encephalitis}

Chi square test for the association of $M B L 2 \mathrm{~A} / \mathrm{O}, \mathrm{H} / \mathrm{L}, \mathrm{P} / \mathrm{Q}$ and $\mathrm{Y} / \mathrm{X}$ alleles, and Fisher exact test for the association of genotypes with the risk of HIVE did not show any significant correlation ( $P \geq 0.05$ [data not shown]). Also, no significant association of MBL2 genotypes with the MBL expression in HIVE, non-HIVE, or HIV negative cases was observed (data not shown).

\section{Discussion}

Almost all the components of classical and alternate pathways of complement activation have been shown to be expressed in the brain, however an MBL mediated complement activation has not been studied in the context of innate immune response in the HIV-1-infected brain. Since MBL can directly interact with HIV-1 by the binding of its carbohydrate recognition domains to the mannose residues present on the glycosylated gp120 or gp41 proteins, its expression in the HIV-1 infected brain can be modulated by the presence of viral proteins. Results from the current study are significant for several reasons.

First, we evaluated the expression of MBL protein in HIV-1 infected post-mortem brain tissues with or without HIV encephalitis and found that MBL was expressed in neurons, astrocytes, microglia, and oligodendrocytes in HIVE emphasizing its underlying importance in the innate immune response to HIV infection in brain.

Second, we observed an increased MBL expression in the neuronal axons of HIVE vs non-HIVE cases implying a potential association of MBL with neuronal injury. The increased MBL expression and deposition in neuronal axons may not only mount an enhanced MBL-mediated complement activation and cytokine response, it can also lead to axonal damage, thus impairing the neuronal function.

Third, using western blots, we confirmed an overall increase in MBL expression in HIVE brain compared with non-HIVE brain implying the presence of functional MBL in HIV-1 infected brain. We observed 3 bands of $32 \mathrm{kDa}$ monomer, $51 \mathrm{kDa}$ dimer, and $78 \mathrm{kDa}$ trimer of MBL. This suggested that the observed expression of MBL was actually reflected in a trimeric functional protein that could lead to increased MBL-mediated complement activation in HIV-1 infected brain.

Fourth, MBL-HIVE link was further confirmed by the presence and co-localization of MCP-1 and MBL in the immunoreactive cells in frontal cortex of the HIVE cases suggesting an MBL-mediated proinflammatory cytokine response and neuroinflammation. MCP-1 is an accepted marker of HIV-1 related neuroinflammation, ${ }^{30}$ and macrophages, endothelial cells, microglia, and astrocytes are known to express MCP-1 against proinflammatory stimuli. ${ }^{31}$ Several cell types expressing chemokine receptor 2 (CCR2, the MCP-1 receptor) such as monocytes, macrophages, basophils, mast cells, T lymphocytes, natural killer cells, and dendritic cells are recruited by MCP-1 to the sites of peripheral or CNS injury. Increased MCP-1 in the CNS has been shown to be strongly associated with HIV-encephalitis and HIV associated dementia. ${ }^{32-39}$ We observed an increased expression of MBL in total brain tissues from HIVE cases compared with non-HIVE or HIV negative cases; however we cannot rule out that an increased infiltration of MBL and/or MCP-1expressing cells in the brain such as monocytes can also lead to increased detection of MBL and MCP-1 through western blots. Nevertheless, our brain cell markers' specific results convincingly show that $\mathrm{MBL}$ is indeed expressed in the HIV-1 infected brain and its overall expression in neuronal axons is increased in the HIV-1 infected brain with encephalitis. Further studies are required to evaluate the potential expression of MBL and MCP-1 from other cell types (eg, monocytes, neutrophils, or $\mathrm{T}$ cells) in the HIV-1 infected brain.

Finally, we confirmed that HIV-1 p24 protein, a marker of HIV-1 replication in the brain, was present at higher levels in the microglia of HIVE cases compared with non-HIVE cases.

Using gp120 transgenic mice, we found that MBL expression did not increase in gp120 transgenic (tg) mice brain compared with normal mice suggesting that other host or viral factors in addition to gp120 may be involved in the induction of MBL expression during HIV-1 neuropathogenesis. However, we cannot rule out an MBLmediated neuroinflammatory response in gp120 mice brain. Also, we studied the brains from 12 month old gp120 tg mice, and it is possible that older mice may show variable expression of MBL. Another possibility is that there may be differential expression of mannose residues in the $\mathrm{N}$-glycans in gp120 expressed in the gp120 tg mice compared with the gp120 expressed in infected microglia in the human brain. This will alter the MBL activation and expression in gp120 mice brain. Further studies are required to evaluate this in gp120 tg mice model.

We did not observe any significant association between the MBL2 genotypes ${ }^{22}$ and the MBL expression or to the 
risk of HIVE in the HIV-1 infected brain. MBL2 genotypes potentially associated with MBL deficiency were present in the brain tissues; however their presence was not significantly associated with altered MBL expression in the studied HIV-1 infected brains with or without HIV encephalitis. Based on our recent findings from large cohorts of HIV-1 infected children ${ }^{22}$ and adults ${ }^{23}$ where we found that the presence of an MBL2 genotype (associated with lower expression of MBL) was linked to the development of neurocognitive impairment, we expected that lower expression of MBL would be associated with the risk of developing HIV encephalitis in the brain, however increased expression of MBL in HIVE cases suggests that chronically activated complement through MBL pathway might contribute to HIV-associated neuroinflammation and neurodegeneration. Enhanced complement synthesis and chronic complement activation have been implicated in the brain damage and progression of several neuroinflammatory and neurodegenerative diseases such as Alzheimer's disease, Huntington's disease or multiple sclerosis. ${ }^{40,41}$ This also suggests that the presence of specific $M B L 2-\mathrm{O} / \mathrm{O}$ variant genotypes may not provide conclusive information about the actual effect of $M B L 2$ gene associated with HIVE. It is possible that the presence of $M B L 2-\mathrm{O} / \mathrm{O}$ or $-\mathrm{X} / \mathrm{X}$ variant genotypes associated with nonfunctional and lower levels of MBL respectively provides a weak innate immune response against the risk of HIV-1 infection. However, expression of MBL is probably enhanced at transcriptional or translational stage once brain cells encounter mannose laden gp120 or gp41 viral proteins. ${ }^{14}$ This in turn potentially leads to over-activation of MBL-mediated complement system and enhanced neuroinflammation in the brain cells. Potential mechanisms of MBL-mediated brain damage may also include opsonization of the surrounding brain cells and phagocytosis by macrophages/microglia, and MBL-dependent bystander lysis following the formation of the membrane attack complex (MAC) that attack neurons. Additionally, MACs might induce the release of arachidonic acid and its metabolites that impart neurological injury. ${ }^{42}$

Although, we did not find any association of MBL2 genotypes with HIV-1 disease progression or CNS impairment by race/ethnicity, ${ }^{22,23}$ a recent report ${ }^{43}$ has shown that increased frequency of $M B L 2-L X / L X$ genotypes was associated with low MBL levels in Euro-derived patients, suggesting a potential role for MBL in the susceptibility to HIV-1 infection in Euro-derived individuals.

Several host and viral factors have been linked to HIV-1 neuropathogenesis and neuroAIDS, ${ }^{44}$ and the role of complement activation in HIV-1 related neuroinflammation and CNS impairment has been suggested. ${ }^{45-47}$ Since HIV infection has been shown to directly induce the expression of complement factors $\mathrm{C} 2$ and $\mathrm{C} 3$ in astrocytes and of $\mathrm{C} 3$ in neurons, ${ }^{48}$ induction of MBL expression in brain through HIV gp120 residues is not unusual. The synthesis of complement factors can be markedly upregulated by inflammatory cytokines, ${ }^{40,41}$ and MBL itself can induce the production of cytokines in brain. ${ }^{49}$ In addition to the release of MBL in plasma by the liver, detectable levels of MBL have been reported in cerebrospinal fluid; however brain tissue-specific enhanced expression of MBL is more likely to correlate with the extent of neuroinflammation and encephalitis in the HIV-1 infected brain.

MBL mediated complement activation is a constituent of innate immune response to the HIV-1; however enhanced MBL synthesis and MBL-mediated complement activation can influence immunological and virological processes in the HIVinfected brain by helping in increased viral spreading within the brain and add to significant neuroinflammation and HIV encephalitis in the terminal stages of neuropathogenesis before death. Indeed, opsonized HIV-1 together with complement receptor-positive cells was shown to enhance cell infection due to improved targeting of the opsonized virions. ${ }^{50-52}$ Also, the opsonization of HIV with MBL and complement fragments may enhance the phagocytosis by astrocytes or microglia. Furthermore, it has been shown that interaction of MBL with HIV-1 is sufficient for virus opsonization. ${ }^{53,54}$

In conclusion, increased MBL expression in the major brain cell types, specifically in the neuronal axons of the HIV encephalitis brain, and higher MCP-1 expression in concurrence with MBL in individuals with HIV encephalitis suggest a potential association of MBL-mediated neuroinflammation and neuronal injury in HIV infected brain. These studies may have implications for observed in-life neurocognitive impairment among HIV-infected individuals and could potentially suggest MBL-based therapeutics against HIV-1 mediated neuroinflammation and neuroAIDS.

\section{Previous presentation in part}

XVIII Conference on Retroviruses and Opportunistic Infections, Boston, MA, February 27-March 3, 2011 (Abstract no. E-142).

\section{Acknowledgments}

The authors would like to acknowledge the support by National Institute of Health (NIH) (MH085608, MH062962) and California HIV/AIDS Research Program IDEA Grant (ID07-SD-160), NIMH Center grant MH62512, California NeuroAIDS Tissue Network (CNTN) U01MH083506, 
R24MH59745 funded under National NeuroAIDS Tissue Consortium (NNTC). Confocal microscopy was done with Olympus FV1000 Microscope at UCSD Neuroscience Microscopy Shared Facility (P30 NS047101).

KKS conceived, designed, coordinated, supervised the study, participated in the immunohistochemistry assays and analyses, and drafted and finalized the manuscript for publication. SN carried out immunofluorescene, confocal microscopy, and western blot assays for human MBL and MCP-1. AA carried out immunohistochemistry in human and mice samples for MBL. TLA was involved in immunohistochemistry and immunofluorescence of MBL in human samples. WD carried out the identification, characterization, and procurement of post-mortem brain tissues. BG carried out the data procurement and data analyses for the demographics and clinical parameters. DJM was involved in the identification of post-mortem brain tissue samples, demographic and clinical data analyses and in drafting the manuscript. EM participated in the design and coordination of the study, in the identification and analyses of post-mortem brain tissue samples, in detailed analyses of immunohistochemistry and immunofluorescence results, and in the manuscript preparation. All the authors have read and approved the text as submitted to the journal.

The San Diego HIV Neurobehavioral Research Center (HNRC) group is affiliated with the University of California, San Diego; the Naval Hospital, San Diego; and the Veterans Affairs San Diego Healthcare System and includes: Director: Igor Grant, MD; Co-Directors: J. Hampton Atkinson, MD, Ronald J. Ellis, MD, PhD, and J. Allen McCutchan, MD; Center Manager: Thomas D. Marcotte, PhD; Jennifer Marquie-Beck, MPH; Melanie Sherman; Neuromedical Component: Ronald J. Ellis, MD, PhD (principal investigator [PI]), J. Allen McCutchan, MD, Scott Letendre, MD, Edmund Capparelli, PharmD, Rachel Schrier, PhD, Terry Alexander, R.N. Debra Rosario, MPH, Shannon LeBlanc; Neurobehavioral Component: Robert K. Heaton, PhD (PI), Steven Paul Woods, PsyD, Mariana Cherner, PhD, David J. Moore, PhD, Matthew Dawson; Neuroimaging Component: Terry Jernigan, PhD (PI), Christine Fennema-Notestine, PhD, Sarah L. Archibald, M.A. John Hesselink, MD, Jacopo Annese, PhD, Michael J. Taylor, PhD; Neurobiology Component: Eliezer Masliah, MD (PI), Cristian Achim, MD, PhD, Ian Everall, FRCPsych, FRCPath, PhD (Consultant); Neurovirology Component: Douglas Richman, MD (PI), David M. Smith, MD; International Component: J. Allen McCutchan, MD (PI); Developmental Component: Cristian Achim, MD, PhD (PI), Stuart Lipton, MD, PhD; Participant Accrual and
Retention Unit: J. Hampton Atkinson, MD (PI), Rodney von Jaeger, M.P.H.; Data Management Unit: Anthony C. Gamst, PhD (PI), Clint Cushman (Data Systems Manager); Statistics Unit: Ian Abramson, PhD (PI), Florin Vaida, PhD, Reena Deutsch, PhD, Anya Umlauf, MS, Tanya Wolfson, MA.

\section{Disclosure}

The authors disclose no conflicts of interest.

\section{References}

1. Garred P, Larsen F, Madsen HO, Koch C. Mannose-binding lectin deficiency - revisited. Mol Immunol. 2003;40(2-4):73-84.

2. Turner MW. The role of mannose-binding lectin in health and disease. Mol Immunol. 2003;40(7):423-429.

3. Kilpatrick DC. Mannan-binding lectin: clinical significance and applications. Biochim Biophys Acta. 2002;1572(2-3):401-413.

4. Thiel S, Vorup-Jensen T, Stover CM, et al. A second serine protease associated with mannan-binding lectin that activates complement. Nature. 1997;386(6624):506-510.

5. Super M, Thiel S, Lu J, Levinsky RJ, Turner MW. Association of low levels of mannan-binding protein with a common defect of opsonisation. Lancet. 1989;2(8674):1236-1239.

6. Valdimarsson H, Stefansson M, Vikingsdottir T, et al. Reconstitution of Opsonizing Activity by Infusion of Mannan-Binding Lectin (MBL) to MBL-Deficient humans. Scand J Immunol. 1998;48(2):116-123.

7. Koch A, Melbye M, Sørensen P, et al. Acute respiratory tract infections and mannose-binding lectin insufficiency during early childhood. JAMA. 2001;285(10):1316-1321.

8. Kakkanaiah VN, Shen GQ, Ojo-Amaize EA, Peter JB. Association of low concentrations of serum mannose-binding protein with recurrent infections in adults. Clin Diagn Lab Immunol. 1998;5(3):319-321.

9. Fidler KJ, Wilson P, Davies JC, Turner MW, Peters MJ, Klein NJ. Increased incidence and severity of the systemic inflammatory response syndrome in patients deficient in mannose-binding lectin. Intensive Care Med. 2004;30(7):1438-1445.

10. Holmskov UL. Collectins and collectin receptors in innate immunity. APMIS. 2000;100(Supp 1):1-59.

11. Phatsara C, Jennen DG, Ponsuksili S, et al. Molecular genetic analysis of porcine mannose-binding lectin genes, MBL1 and MBL2, and their association with complement activity. Int J Immunogenet. 2007;34(1): 55-63.

12. Wagner S, Lynch NJ, Walter W, Schwaeble WJ, Loos M. Differential expression of the murine mannose-binding lectins $\mathrm{A}$ and $\mathrm{C}$ in lymphoid and nonlymphoid organs and tissues. J Immunol. 2003;170(3): 1462-1465.

13. Zanetta JP. Mannose-binding lectins in cerebrum development. Prog Mol Subcell Biol. 2003;32:75-96.

14. Ji X, Gewurz H, Spear GT. Mannose binding lectin (MBL) and HIV. Mol Immunol. 2005;42(2):145-152.

15. Jacobs A, Hartman K, Laue T, Caffrey M. Sedimentation velocity studies of the high-molecular weight aggregates of the HIV gp41 ectodomain. Protein Sc. 2004;13(10):2811-2813.

16. Falangola MF, Castro-Filho BG, Petito CK. Immune complex deposition in the choroid plexus of patients with acquired immunodeficiency syndrome. Ann Neurol. 1994;36(3):437-440.

17. Jack DL, Dodds AW, Anwar N, et al. Activation of complement by mannose-binding lectin on isogenic mutants of Neisseria meningitidis serogroup B. J Immunol. 1998;160:1346-1353.

18. Hibberd ML, Sumiya M, Summerhill SA, Booy R, Levin M. Association of variants of the gene for mannose binding lectin with susceptibility to meningococcal disease. Lancet. 1999;353(9158):1049-1053.

19. Gadjeva M, Paludan SR, Thiel S, et al. Mannan-binding lectin modulates the response to HSV-2 infection. Clin Exp Immunol. 2004;138(2):304-311. 
20. Heggelund L, Mollnes TE, Ueland T, Christophersen B, Aukrust P, Froland SS. Mannose-binding lectin in HIV infection: relation to disease progression and highly active antiretroviral therapy. $J$ Acquir Immune Defic Syndr. 2003;32:354-361.

21. Garred P, Madsen HO, Balslev U, et al. Susceptibility to HIV infection and progression of AIDS in relation to variant alleles of mannosebinding lectin. Lancet. 1997;349(9047):236-240.

22. Singh KK, Lieser A, Ruan PK, Fenton T, Spector SA. An age-dependent association of mannose-binding lectin-2 genetic variants on HIV-1-related disease in children. J Allergy Clin Immunol. 2008;122(1):173-180.

23. Spector SA, Singh KK, Gupta S, et al. APOE [epsilon] and MBL-2 $\mathrm{O} / \mathrm{O}$ genotypes are associated with neurocognitive impairment in HIVinfected plasma donors. AIDS. 2010;24(10):1471-1479.

24. Heaton R, Grant I, Butters N, et al. The HNRC 500-neuropsychology of HIV infection at different disease stages. $J$ Int Neuropsychol Soc. 1995;1(3):231-251.

25. Achim C, Wang R, Miners D, Wiley C. Brain viral burden in HIV infection. J Neuropathol Exp Neurol. 1994;53(3):284-294.

26. Masliah E, Achim C, Ge N, De Teresa R, Wiley CA. Cellular neuropathology in HIV encephalitis. Res Publ Assoc Res Nerv Ment Dis. 1994; 72:119-131.

27. Masliah E, Heaton R, Marcotte T, et al. Dendritic injury is a pathological substrate for human immunodeficiency virus-related cognitive disorders. Ann Neurol. 1997;42(6):963-972.

28. Sanders V, Everall IP, Johnson RW, Masliah E, Group HNRC. Fibroblast growth factor modulates HIV co-receptor expression by neural cells. J Neurosci Res. 2000;59(5):671-679.

29. Toggas SM, Masliah E, Rockenstein EM, Rall GF, Abraham CR, Mucke L. Central nervous system damage produced by expression of the HIV-1 coat protein gp120 in transgenic mice. Nature. 1994;367(6459): 188-193.

30. Dhillon NK, Williams R, Callen S, Zien C, Narayan O, Buch S. Roles of MCP-1 in development of HIV-dementia. Front Biosci. 2008;13: 3913-3918.

31. Gu L, Rutledge B, Fiorillo J, et al. In vivo properties of monocyte chemoattractant protein-1. J Leukoc Biol. 1997;62(5):577-580.

32. Cinque P, Vago L, Mengozzi M, et al. Elevated cerebrospinal fluid levels of monocyte chemotactic protein-1 correlate with HIV-1 encephalitis and local viral replication. AIDS. 1998;12(11):1327-1332.

33. Conant K, Garzino-Demo A, Nath A, et al. Induction of monocyte chemoattractant protein-1 in HIV-1 Tat-stimulated astrocytes and elevation in AIDS dementia. Proc Natl Acad Sci U S A. 1998;95(6): 3117-3121.

34. Kelder W, McArthur JC, Nance-Sproson T, McClernon D, Griffin DE. Beta-chemokines MCP-1 and RANTES are selectively increased in cerebrospinal fluid of patients with human immunodeficiency virusassociated dementia. Ann Neurol. 1998;44(5):831-835.

35. Sozzani S, Introna M, Bernasconi S, et al. MCP-1 and CCR2 in HIV infection: regulation of agonist and receptor expression. J Leukoc Biol. 1997;62(1):30-33.

36. Zink MC, Coleman GD, Mankowski JL, et al. Increased macrophage chemoattractant protein-1 in cerebrospinal fluid precedes and predicts simian immunodeficiency virus encephalitis. $J$ Infect Dis. 2001;184(8):1015-1021.

37. Sanders VJ, Pittman CA, White MG, Wang G, Wiley CA, Achim CL. Chemokines and receptors in HIV encephalitis. AIDS. 1998;12(9): 1021-1026.

Neurobehavioral HIV Medicine

\section{Publish your work in this journal}

Neurobehavioral HIV Medicine is an international, peer-reviewed, open access journal focusing on advances in research in HIV/ AIDS, with specific reference to the neurological, psychiatric and behavioral consequences of the disease, concomitant infections and specific antiretroviral therapy. The manuscript
38. Mengozzi M, De Filippi C, Transidico P, et al. Human immunodeficiency virus replication induces monocyte chemotactic protein-1 in human macrophages and U937 promonocytic cells. Blood. 1999;93(6):1851-1857.

39. Hicks A, Potula R, Sui YJ, et al. Neuropathogenesis of lentiviral infection in macaques: roles of CXCR4 and CCR5 viruses and interleukin-4 in enhancing monocyte chemoattractant protein-1 production in macrophages. Am J Pathol. 2002;161(3):813-822.

40. Speth C, Schabetsberger T, Mohsenipour I, et al. Mechanism of human immunodeficiency virus-induced complement expression in astrocytes and neurons. J Virol. 2002;76(7):3179-3188.

41. Gasque P, Dean YD, McGreal EP, VanBeek J, Morgan BP. Complement components of the innate immune system in health and disease in the CNS. J Immunopharmacology. 2000;49(1-2):171-186.

42. Power C, Johnson RT. HIV-1 associated dementia: clinical features and pathogenesis. Can J Neurol Sci. 1995;22(2):92-100.

43. Da Silva GK, Guimarães R, Mattevi VS, et al. The role of mannosebinding lectin gene polymorphisms in susceptibility to HIV-1 infection in Southern Brazilian patients. AIDS. 2011;25(4):411-418.

44. Shapshak P, Kangueane P, Fujimura RK, et al. Editorial neuroAIDS review. AIDS. 2011;25(2):123-141.

45. Datta PK, Rappaport J. HIV and complement: hijacking an immune defense. Biomed Pharmacother. 2006;60(9):561-568.

46. Jongen PJ, Doesburg WH, Ibrahim-Stappers JL, Lemmens WA, Hommes OR, Lamers KJ. Cerebrospinal fluid $\mathrm{C} 3$ and $\mathrm{C} 4$ indexes in immunological disorders of the central nervous system. Acta Neurol Scand. 2000;101(2):116-121.

47. Speth C, Dierich MP, Sopper S. HIV-infection of the central nervous system: the tightrope walk of innate immunity. Mol Immunol. 2005;42(2): 213-228.

48. Speth C, Stöckl G, Mohsenipour I, et al. Human immunodeficiency virus type 1 induces expression of complement factors in human astrocytes. J Virol. 2001;75(6):2604-2615.

49. Heggelund L, Mollnes TE, Espevik T, et al. Modulatory effect of mannose-binding lectin on cytokine responses: possible roles in HIV infection. Eur J Clin Invest. 2005;35(12):765-770.

50. Reisinger EC, Vogetseder W, Berzow D, et al. Complement-mediated enhancement of HIV-1 infection of the monoblastoid cell line U937. AIDS. 1990;4:961-965.

51. Thieblemont N, Haeffner-Cavaillon N, Haeffner A, Cholley B, Weiss L, Kazatchkine MD. Triggering of complement receptors CR1 (CD35) and CR3 (CD11b/CD18) induces nuclear translocation of NF-kappa B (p50/ p65) in human monocytes and enhances viral replication in HIV-infected monocytic cells. J Immunol. 1995;155(10):4861-4867.

52. Boyer V, Desgranges C, Trabaud MA, Fischer E, Kazatchkine MD. Complement mediates human immunodeficiency virus type 1 infection of a human T cell line in a CD4- and antibody-independent fashion. J Exp Med. 1991;173(5):1151-1158.

53. Saifuddin M, Hart ML, Gewurz H, Zhang Y, Spear GT. Interaction of mannose-binding lectin with primary isolates of human immunodeficiency virus type 1. J Gen Virol. 2000;81(Pt 4):949-955.

54. Ying $\mathrm{H}, \mathrm{Ji} X$, Hart ML, et al. Interaction of mannose-binding lectin with HIV type 1 is sufficient for virus opsonization but not neutralization. AIDS Res Hum Retroviruses. 2004;20(3):327-335.

management system is completely online and includes a very quick and fair peer-review system, which is all easy to use. Visit http://www.dovepress.com/testimonials.php to read real quotes from published authors. 